\title{
Vom Nationalitätenministerium bis zur Vertreibung

\author{
Die rechtshistorische Aufarbeitung der Ungarndeutschen \\ in der ersten Hälfte des 20. Jahrhunderts
}

\begin{abstract}
From the Ministry of Ethnic Affairs to the expulsion of the German Minority. The fate of the Germans in Hungary in the first half of the $20^{\text {th }}$ century from a legal historical perspective.

The topic of this article is the history of the German minority in Hungary in the first half of 20 th century from the point of view of the legal history. After the First World War, Hungary became a nation state, in which the approximately half a million Danube Swabians were the biggest minority. Directly after the First World War, Hungary tried to implement another minority policy than at the time of the Austro-Hungarian Monarchy, and concepts of autonomy were made. This period ended after the peace treaty of Trianon, and the so-called Horthy regime was characterised by the return to the traditions of the period of the dual monarchy. This changed before the Second World War, as Nazi Germany made the German minorities abroad an instrument of its politics. The Germans founded the Volksbund, a national-socialistic oriented ethnic organisation, Germans got cultural autonomy but were forced to enlist in the German armed forces. After the Second World War, the deprivation of rights of the Germans begun: in 1945-1946, 60,000 were transported to the Soviet Union for forced labour; in 1946-1948 approximately 166,000 were forced to leave Hungary, and the 200,000 left in Hungary had no citizens' rights at all.
\end{abstract}

Keywords: Danube Swabians - forced migration - German minority - minority rights

\section{Die Vorgeschichte}

In den letzten Jahrzehnten der ÖsterreichischUngarischen Monarchie lebten auf dem Gebiet des Königreiches Ungarn 1,99 Millionen Deutsche - Tendenz leicht rückläufig, um die Jahrhundertwende verloren die Deutschen ihre Stellung als zweitgrößte Minderheit an die Slowaken. ${ }^{1}$ Die Deutschen galten im von Nationalitätenkonflikten geprägten Land als die "gute Minderheit" - da sie kaum Forderungen an kollektiven Rechten formulierten, über keine landesweite politische oder gesellschaftliche Organisation verfügten. Dieser Umstand ist damit zu

\footnotetext{
${ }^{1}$ KatUS, Nemzetiségi adatsorok 195.
}

erklären, dass die Deutschen nicht in einem einheitlichen, zusammenhängenden Siedlungsgebiet lebten, zudem waren die Angehörigen der deutschen Minderheit hinsichtlich der Abstammung sehr vielfältig. ${ }^{2}$ Große Vielfalt existierte auch in der Religion, in der ökonomischen sowie sozialen Situation, woraus sich auch sehr unterschiedliche Interessen und grundlegend unterschiedliche Möglichkeiten der Vertretung der eigenen Interessen ergaben.

\footnotetext{
${ }^{2}$ SEEWANN, Geschichte 2, 92.
} 


\section{Die Regelung der Rechts- stellung der Ungarndeutschen}

Den Zeitraum meiner Untersuchungen habe ich in vier Epochen unterteilt, die erste ist die Zeit des Versuchs einer beispielhaften Nationalitätenpolitik unmittelbar nach dem ersten Weltkrieg (1918-1920), die zweite ist von der Rückkehr zum Erbe der Donaumonarchie in der Nationalitätenpolitik gekennzeichnet (1920-1938), die dritte Periode 1938-1945, in der je nach Sichtweise eine privilegierte Stellung oder die Verbesserung der rechtlichen Lage der ungarndeutschen Bevölkerung zu verzeichnen war tatsächlich haben die Kriegsereignisse immer stärkeren Einfluss genommen. Schließlich begann ab 1945 die Epoche der Entrechtung, die de jure bis 1950 dauerte und auch die Vertreibung von etwa der Hälfte der Ungarndeutschen 19461948 mit beinhaltete.

Die Entwicklung war also nicht linear, und allgemeine Aussagen und Tendenzen über einen Zeitraum von 50 Jahren sind schwer festzustellen. Für die rechtliche Regelung war aber während der ganzen Epoche bezeichnend, dass Parlamentsgesetze eher die Ausnahme waren. Vielmehr wurden die Rechte der nationalen Minderheiten in niederrangigen Rechtsnormen, wie Regierungs- oder Ministerialverordnungen geregelt. Oft sind wichtige Vorschriften in Pseudonormen ausgelagert worden, wie Weisungen, Bekanntmachungen, interne Vorschriften, oder sogar Rundbriefe, die zwar einen normativen Charakter hatten, jedoch formlos herausgegeben worden sind, und für die Betroffenen nicht zugänglich waren. Die politischen Ziele und Intentionen hinter den verschiedenen Rechtsnormen waren von Epoche $\mathrm{zu}$ Epoche, teilweise auch innerhalb einer Epoche, unterschiedlich und beeinflussten die Gesetzgebung maßgeblich. Die erzielten Ergebnisse hingen von der Einstellung sowie Durchsetzungskraft der Verwaltung ab, es gab viele Regelungen, die nur auf dem Papier bestanden, und keine tatsächliche Wirkung ent- falten konnten - mangels Zeit, oder mangels Willens und Möglichkeiten der Durchsetzung.

\section{Der Versuch einer beispiel- haften Nationalitätenpolitik} Territoriale und kulturelle Autonomiekonzepte

Die Zeit nach dem ersten Weltkrieg ist in Ungarn von tiefgreifenden Veränderungen und einer äußerst instabilen und wechselhaften politischen Lage gekennzeichnet. Die aufeinander folgenden politischen Regime - Erste Republik, Räterepublik, anfängliche Horthy-Ära - verfolgten verschieden Ansätze und Konzepte der Nationalitätenpolitik, nur ihr Ziel war gleich: die territoriale Integrität von Ungarn möglichst zu wahren.

Die Erste Ungarische Republik (die sich als Ungarische Volksrepublik bezeichnete) war von den 14 Punkten des US-amerikanischen Präsidenten Wilson beeinflusst und folglich dem Selbstbestimmungsrecht der Völker verschrieben. ${ }^{3}$ Entsprechend erfolgte eine Abkehr von dem Konzept der „einzigen politischen Nation“ der Dualismus-Zeit, die eine Hegemonie- und Vormachtstellung des ungarischen Volkes in Ungarn postulierte. ${ }^{4}$ Etwas idealistisch ging man davon aus, dass die Auflösungserscheinungen der Monarchie und die Unabhängigkeitsbestrebungen der Nationalitäten aufgehalten werden könnten. Juristisch erfasst wurden diese Gedanken hinsichtlich der deutschen Minderheit im Volksgesetz Nr.6 über die Ausübung des Selbstbestimmungsrechts des ungarndeutschen Volkes. Erlassen am 28. Januar 1919, hat das Gesetz die Deutschen als Nation anerkannt, ihnen eine breite kulturelle Autonomie und in den geschlossenen Siedlungsgebieten auch eine territoriale Autonomie zugestanden. Es sollte eine eigene Deutsche Nationalversammlung gewählt werden, sowie

\footnotetext{
${ }^{3}$ FISCHER, Oszkár Jászi und Mihály Károlyi 66-80.

${ }^{4}$ SCHLETT, Eötvös József 267.
} 
eine anteilige Repräsentation der Deutschen in der ungarischen Nationalversammlung erfolgen, ein Landesregierungsausschuss für die Deutschen in Ungarn gebildet und ein deutscher Minister in die ungarische Regierung ernannt werden. ${ }^{5}$ Ein provisorischer Landesregierungsausschuss wurde mit der Leitung von Dr. Guido Gündisch gebildet und Johann/János Junker wurde zum deutschen Minister ernannt, aber ansonsten konnten die Vorschriften des Gesetzes nicht vollzogen werden. ${ }^{6}$

Nicht zuletzt, weil am 21. März 1919 die Ungarische Räterepublik entstand, die sich als kommunistischer und föderaler Staat verstand, wie die Verfassung vom 23. Juni 1919 darlegt. ${ }^{7}$ Da zu diesem Zeitpunkt das historische Staatsgebiet bereits zerfallen war, bedeutete es praktisch eine deutsche und ruthenische Autonomie. Die Deutschen erhielten ein eigenes Deutsches Volksamt, Henrik Kalmár, ein deutschstämmiger Sozialdemokrat, wurde Volkskommissar. Es wurde freier Sprachgebrauch zugesichert, ein Deutscher Kulturbund für Ungarn gegründet, es wurde versprochen, 450 deutsche Schulen zu errichten und drei autonome Bezirke der Deutschen aufzustellen, West mit Sopron, Mitte mit Budapest und Süd mit Bonyhád als Sitz. ${ }^{8}$ Die stets umkämpfte Räterepublik konnte diese Versprechen jedoch nicht erfüllen - da sie nur 133 Tage lang bestand.

Nach Fall der Räterepublik und Einmarsch der rumänischen Truppen begann eine Konsolidierung der Lage und Miklós Horthy, der spätere Reichsverweser, übernahm faktisch die Macht. Da es zu dieser Zeit bereits als sicher galt, dass das gesamte historische Staatsgebiet Ungarns nicht mehr zu halten war, war nunmehr das Ziel, möglichst günstige Verhandlungspositionen bei den Friedensverhandlungen zu erzielen.

\footnotetext{
${ }^{5}$ BELLÉR, A Magyar Népköztársaság 5-6.

${ }^{6}$ SEEWANN, Geschichte 2, 184.

${ }^{7}$ BELLÉR, A Magyar Népköztársaság 8.

${ }^{8}$ Ebd. 12.
}

In diesem Sinne wurde das so genannte Nationalitätenministerium gegründet - rechtlich gesehen wurde ein für die nationalen Minderheiten zuständiger Minister ohne Geschäftsbereich ernannt, der jedoch über ein eigenes Amt verfügte. Zum Minister wurde am 15. August 1919 Prof. Jakob Bleyer, ein Germanistikprofessor ungarndeutscher Abstammung, ernannt. ${ }^{9}$ Er war persönlich zwar dem früheren ungarischen Staatskonzept verschrieben, sollte jedoch in seiner Position für die territoriale Autonomie von Deutsch-Westungarn (dem heutigen Burgenland) werben, um dieses Gebiet für Ungarn erhalten zu können. ${ }^{10}$ Es wurde auch ein neues Regelwerk über die Rechte der nationalen Minderheiten erlassen, die Regierungsverordnung 4044/1919. M.E., die als Gleichberechtigungsverordnung bekannt wurde. In der Verordnung ist zwar keine Rede mehr von einer einheitlichen ungarischen politischen Nation, es stand aber dennoch teilweise im Sinne des Nationalitätengesetzes von 1868, es sah nur - wenn auch breit gefasste - individuelle Rechte vor, vor allem auf Gebrauch der Muttersprache und Schulunterricht in der Muttersprache. ${ }^{11} \mathrm{Ob}$ jemals politischer Wille zum tatsächlichen Vollzug dieses Regelwerkes (mit den ergänzenden Ministerialverordnungen) bestand, kann wohl nicht abschließend beantwortet werden. Denn diese vergleichsweise liberale Nationalitätenpolitik und Gesetzgebung nahm schnell ein Ende, als deren Erfolglosigkeit - die Friedensverhandlungen konnten nicht wie erwünscht beeinflusst werden - klar wurde.

\footnotetext{
${ }^{9}$ VITÁRI, Új kisebbségpolitika ellenérzésekkel 193.

${ }^{10}$ TILKOVSZKY, A Bleyer-portré problematikus vonásai 261.

11 SEEWANN, Geschichte 2, 186.
} 


\section{Rückkehr zum}

\section{Erbe der Monarchie}

\section{Nationalitätengesetzgebung in der Horthy-Ära}

Mit dem Friedensvertrag von Trianon am 4. Juni 1920 verlor Ungarn fast zwei Drittel seines Staatsgebietes an die Nachbarstaaten, und aus dem Vielvölkerstaat Ungarn wurde ein Nationalstaat, 89 \% der Bevölkerung hatten Ungarisch als Muttersprache, die Deutschen bildeten mit etwa 550.000 Personen die größte nationale Minderheit. ${ }^{12}$

Die als Zugeständnisse aufgefassten Rechte, die weiter gingen als jene in der Zeit des Dualismus, wurden schnell zurückgenommen, allgemein herrschte ein "nationalitätenfeindliches" Klima, obwohl faktisch kaum mehr nationale Minderheiten in Ungarn lebten - sie wurden ja für den Zerfall des Landes verantwortlich gemacht. ${ }^{13}$ Das spiegelte sich schnell in der Staatsorganisation und in der Gesetzeslage wider. Das Nationalitätenministerium wurde aufgelöst, nachdem Bleyer am 16. Dezember 1920 aus der Regierung ausschied, ${ }^{14}$ und auch die liberale Gleichberechtigungsverordnung wurde zurückgenommen. Die Rechte der Nationalitäten wurden mit der Regierungsverordnung 4800/1923. M.E. neu geregelt. Sie war formell die Vollzugsverordnung zum Friedensvertrag von Trianon; die dort normierten völkerrechtlichen Verpflichtungen Ungarns wurden damit ins ungarische Recht inkorporiert. ${ }^{15}$ Sie gestattete den Sprachgebrauch auf lokaler Ebene, wenn ein Fünftel der Bevölkerung zu einem der Minderheiten gehörte, jedoch konnten Anträge und Eingaben bei der zuständigen Behörde in der Muttersprache eingereicht werden - und mussten auch so beantwortet werden. Das Recht zur Schulbildung in der Muttersprache wurde zugestanden, die Verordnung

\footnotetext{
12 SEIDE, Die Deutschen in Ungarn 148.

${ }^{13}$ SeEWANN, Geschichte 2, 187.

${ }^{14}$ BELLÉR, Az ellenforradalmi 1308.

15 SEIDE, Die Deutschen in Ungarn 151.
}

des Ministers für Bildung und Kultus 110.478/1923 VKM regelte drei Typen der Nationalitätenschulen: Typ „A $\mathrm{A}^{\prime}$ hatte die Minderheitensprache als Unterrichtssprache, die ungarische Sprache wurde als Lehrfach unterrichtet, in den Schulen Typ „B“ erfolgte der Unterricht zweisprachig, bei dem Typ "C" war Ungarisch die Unterrichtssprache, die Nationalitätensprache wurde lediglich als Lehrfach unterrichtet. ${ }^{16}$ Die Entscheidung darüber sollte eine eigens dafür einberufene Elternkonferenz treffen, jedoch konnte diese Entscheidung vom Schulträger revidiert werden - entsprechend wenig Schulen von Typ "A“ und "B“ wurden errichtet, ${ }^{17}$ weiterführende Schulen mit deutscher Unterrichtssprache fehlten komplett. ${ }^{18}$

Trotz der im Vergleich zu der Ära der Doppelmonarchie kaum verbesserten Lage gab es von der Seite der ungarndeutschen Gemeinschaft weiterhin keine politischen Forderungen - in dieser Hinsicht blieben sie weiterhin die "gute Minderheit". Der von der Neuausrichtung der Nationalitätenpolitik enttäuschte Jakob Bleyer trat weiterhin für die Ungarndeutschen ein, er gründete 1921 eine Wochenzeitung, das „Sonntagsblatt für das deutsche Volk in Ungarn",19 und 1923 die erste landesweite Organisation der Ungarndeutschen, den Ungarländischen Deutschen Volksbildungsverein. ${ }^{20}$

\section{Privilegierte (Rechts)Stellung? Die Ungarndeutschen im Schatten des Dritten Reiches}

Ab Mitte der 1930er Jahre erfolgten Veränderungen in der Lage der Ungarndeutschen, die vorerst noch nicht in der Gesetzgebung ihre

\footnotetext{
16 Ebd. 153.

17 SEEWANN, Geschichte 2, 240.

${ }^{18}$ BLEYER, A hazai német kisebbség kérdéséről 74.

${ }^{19}$ TILKOvsZKY, Német nemzetiség 46.

${ }^{20} \mathrm{Zu}$ Lebenswerk Bleyers: HAMBUCH, Jakob Bleyer; SEIDE, Die Deutschen in Ungarn 156.
} 
Abdrücke fanden, aber tiefgreifende Prozesse in Gang brachten. Zum einen stieg nach der Machtübernahme der Nationalsozialisten im Deutschen Reich 1933 das Interesse am ausländischen Deutschtum - auch als politisches Instrument gegen die jeweiligen Heimatstaaten. ${ }^{21}$ Gleichzeitig vollzog sich ein Generationswechsel in der ungarndeutschen Elite, der zugleich ein Streit um das Erbe des 1933 verstorbenen Bleyer bedeutete, ${ }^{22}$ und einen Richtungswechsel mit sich zog. Es trat eine Gruppe - teilweise in Deutschland ausgebildeter - junger Akademiker in Erscheinung, die eher der völkischen Richtung zuzuordnen waren, ${ }^{23}$ und sich in der Volksdeutschen Kameradschaft organisierten. ${ }^{24}$ Auf Druck des Dritten Reiches wurde 1938 der Volksbund der Deutschen in Ungarn als Volksgruppenorganisation gegründet, wobei der nationalsozialistische Charakter damals noch verborgen blieb. ${ }^{25}$

Eine Änderung in der Rechtstellung der deutschen Minderheit in Ungarn bedeutete das Volksgruppenabkommen, abgeschlossen am 30. August 1940 (quasi als „Gegenleistung“ von Ungarn für den sogenannten zweiten Wiener Schiedsspruch). Es sicherte den Deutschen in Ungarn ein eigenes Schulwesen zu, ermöglichte die Gründung von eigenen gesellschaftlichen und wirtschaftlichen Organisationen, einen ungehinderten Kontakt mit Deutschland, sowie ein freies Bekenntnis zum nationalsozialistischen Gedankengut und verbot jegliche Maßnahmen zur Madjarisierung. ${ }^{26}$ Entgegen den deutschen Bestrebungen war die ungarische Regierung nicht bereit, der Organisation einen öffentlichrechtlichen Status zu gewähren, und weigerte

\footnotetext{
${ }^{21}$ Dazu ausführlich: TILKOVSZKY, Teufelskreis.

22 Nachruf: GRATZ, 12-16.

${ }^{23}$ RozS, A népinémet mozgalom erősödése 399.

24 SPANNENBERGER, Nemzetiszocialista népcsoportpolitika 428.

${ }^{25}$ Ausführlich dazu: SPANNENBERGER, A magyarországi Volksbund.

${ }^{26}$ BALOGH, SIPOS, KARDOS, A magyar állam 295-296.
}

sich auch, das Volksgruppenabkommen - das als völkerrechtlicher Vertrag abgeschlossen wurde - in einem Gesetz zu verkünden. Das erfolgte schließlich mit der Regierungsverordnung 8490/1940 ME. ${ }^{27}$

Die durchaus zu verzeichnenden positiven Veränderungen - es wurden deutschsprachige Schulen, auch Gymnasien errichtet - waren dadurch überschattet, dass Deutschland die Ungarndeutschen als zusätzliche Ressource betrachtete. Die ungarische Regierung willigte mit Notenwechsel zu den Rekrutierungen zur Waffen-SS $1942^{28}$ und $1943^{29}$ ein, nach der deutschen Besatzung erfolgte dies nicht mehr auf freiwilliger Basis, laut des Notenwechsels vom 14. April 1944 konnten Ungarndeutsche zu den deutschen Streitkräften zwangsverpflichtet werden. ${ }^{30}$

Es ist anzumerken, dass, genauso wie die vorherigen Regelungen, auch das Volksgruppenabkommen und die Rekrutierungsabkommen nicht komplett umgesetzt wurden, die ungarische Verwaltung zeigte auch in diesem Fall wenig Eifer. Vor allem die Rekrutierung zur Waffen-SS wurde bewusst und gezielt behindert, ${ }^{31}$ oft auf Wunsch der Betroffenen. Eine Vielzahl der Ungarndeutschen war den nationalsozialistischen Gedanken, soweit sie ihr überhaupt bekannt waren, eher abgeneigt und betrachtete Ungarn als ihre Heimat.

\section{Die Entrechtung und Ver- treibung der Ungarndeutschen}

Die die deutsche Minderheit schwer benachteiligenden Maßnahmen begannen bereits am 22. Dezember 1944 als der Befehl Nr. 0060 der

\footnotetext{
27 SEEWANN, Geschichte 2, 326.

28 TILKOVSZKY, SS-toborzás Magyarországon 39.

${ }^{29}$ Ebd. 136.

30 SPANNENBERGER, A magyarországi Volksbund 362.

${ }^{31}$ FÜZES, ÚJVÁRI, Mentsvár 43.
} 
Roten Armee ausgegeben wurde. ${ }^{32}$ Er verpflichtete alle Deutschen, Arbeitsdienst in unmittelbarer Frontnähe $\mathrm{zu}$ leisten, tatsächlich wurden aber aufgrund dieses Befehls etwa 60.000-65.000 Deutsche in die Sowjetunion verschleppt, wo sie jahrelang Zwangsarbeit leisten mussten. ${ }^{33}$ Die so genannte "Malenkij Robot" ("malenkaja rabota“, Russisch für „ein wenig Arbeit“) entbehrte jeglicher Rechtsgrundlage im ungarischen Recht, die ungarische Provisorische Nationalregierung war aber nicht in der Lage, den eigenen Staatsangehörigen Schutz zu leisten. ${ }^{34}$

Die Benachteiligung der Ungarndeutschen begann formal mit der Bodenreform, verkündet am 15. März 1945, die die Konfiszierung des Grundbesitzes von Landesverrätern, Volksbundmitgliedern und Kriegs- und volksfeindlichen Verbrechern vorschrieb. Als Landesverräter, kriegs- und volksfeindlicher Verbrecher war anzusehen, "[...] der nach freiwilliger Meldung in einer deutschen faschistischen Militär- oder Ordnungseinheit eingetreten ist; [...], der seinen deutsch klingenden Familiennamen wieder angenommen hat. ${ }^{\prime 35}$ Vor allem eine vermutete Volksbundmitgliedschaft - die oft nicht bewiesen werden konnte - führte dazu, dass vielen ungarndeutschen Familien ihre Lebensgrundlage entzogen wurde. ${ }^{36}$

Die Ungarndeutschen wurden auch vom Wahlrecht ausgeschlossen, das Gesetz Nr. 8 von 1945 schloss von dem ansonsten allgemeinen Wahlrecht all diejenigen aus,37 deren Grundbesitz konfisziert wurde, die Mitglieder, Anführer oder Unterstützer des Volksbundes oder der Hitlerjugend waren, sowie alle, die sich zur

\footnotetext{
32 ZIELBAUER, Verschleppung 1944/45 10.

${ }^{33}$ FÜZES, Modern rabszolgaság 21.

${ }^{34}$ STARK, Malenki robot 75.

${ }^{35}$ Verordnung 600/1945. ME. § 5 (Zur Gesetzeskraft erhoben mit dem Gesetz Nr. 6 von 1945).

${ }^{36}$ TóTH, Földreform 5018.

${ }^{37}$ MeZEY, Magyar alkotmánytörténet 189.
}

deutschen Nationalität bekannt haben - praktisch also sämtliche Ungarndeutschen. ${ }^{38}$

Die Vertreibung der Deutschen aus Ungarn erfolgte in einem formal juristisch geregelten Prozess, eine „wilde Vertreibung“" gab es nicht. ${ }^{39}$ Die Regelung erfolgte in Regierungs- und Ministerialverordnungen, die eine Besonderheit gegenüber ähnlichen Rechtsnormen in anderen Staaten der Region haben: sie verzichteten bewusst auf den Begriff „kollektive Schuld“, um den Nachbarstaaten, die gegenüber der dortigen ungarischen Bevölkerung ähnliche Maßnahmen getroffen haben, keine Argumente zu liefern; der Effekt war aber damit der gleiche. ${ }^{40}$ Das Regelwerk war komplex, richtete sich aber praktisch gegen alle Ungarndeutschen. Die wichtigste Norm war die Regierungsverordnung 12.3300/1945 ME., verkündet am 29. Dezember 1945, die als "Aussiedlungsverordnung“ bekannt wurde. §1 lautete: „Nach Deutschland überzusiedeln verpflichtet ist derjenige ungarische Staatsbürger, der bei der letzten Volkszählung sich zur deutschen Nationalität oder deutschen Muttersprache bekannt hat, oder seinen madjarisierten Namen in einen deutsch klingenden geändert hat, der Mitglied des Volksbundes oder irgendeiner deutschen bewaffneten Einheit war." Demnach sollten alle Deutschen Ungarn verlassen, die Maßnahmen wurden in Verordnungen des Innenministers konkretisiert. ${ }^{41}$ Es erfolgten zahlreiche Änderungen, die den Kreis der Betroffenen verringerten, so wurden diejenige ex lege ausgenommen, die als Nationalität bei der Volkszahlung 1941 Ungarisch angegeben hatten. ${ }^{42}$

Letztendlich wurden etwa 166.000 Ungarndeutsche vertrieben, 1946 in die amerikanische, 1947-48 in die sowjetische Besatzungszone, wei-

\footnotetext{
${ }^{38}$ Gesetz Nr. 8 von $1945 \S 5$.

39 SEEWANN, A németek Kelet-Európából 349.

${ }^{40}$ ROMSICS, Az 1947-es párizsi békeszerződés 128.

${ }^{41}$ Verordnung des Innenministers 70.010/1946. BM.

42 Verordnung 12.200/1947. Korm.
} 
tere 80.000 waren nach Westen geflüchtet, in die Sowjetunion verschleppt oder galten als verschollen. ${ }^{43}$ Dass nicht alle Deutschen aus Ungarn vertrieben wurden, lag nicht an der ungarischen Gesetzgebung oder Verwaltung, sondern daran, dass die Besatzungsmächte in Deutschland nicht in der Lage waren, weitere Vertriebene aus Ungarn aufzunehmen. ${ }^{44}$

Die formelle rechtliche Benachteiligung der Deutschen in Ungarn dauerte bis 1950, mit der Verordnung des Ministerrates 84/1950 MT. wurde normiert, dass die sich in Ungarn aufhaltenden Deutschen ,ungarische Staatsbürger und mit den anderen in aller Hinsichten gleichberechtigten Bürger der Volksrepublik Ungarn“" seien, und die vorherigen restriktiven Vorschriften nicht mehr anzuwenden seien, die bereits eingetretenen Rechtsfolgen aber unberührt bleiben. ${ }^{45}$ Die Ungarndeutschen erfuhren weiterhin Nachteile, deutschen Schulunterricht gab es bis 1952 nicht, ${ }^{46}$ ein landesweiter Dachverband konnte erst 1955 gegründet werden. ${ }^{47}$ So ist es nicht verwunderlich, dass sich bei der Volkszählung 1949 nur 2600 Personen zur deutschen Nationalität bekannt haben. ${ }^{48}$

\section{Juristische Aufarbeitung des Schicksals der Ungarndeutschen}

Die politische und juristische Aufarbeitung der Entrechtung begann in den 1980er Jahren, und beschleunigte sich mit der politischen Wende 1989-90. Noch vor den ersten demokratischen Wahlen, im Frühjahr 1990 erklärte das ungarische Parlament die Verschleppung und Ver-

\footnotetext{
${ }^{43}$ CZiBUlKa, HeinZ, LAKATOS, A magyarországi németek 183 .

${ }^{4}$ SeEWANN, Geschichte 2,349; TóTH, Telepítések Magyarországon 177.

${ }^{45}$ Verordnung 84/1950. MT. § 3 Abs. 1.

${ }^{46}$ FÖGLEIN, Etnicum és educatio 92.

47 SeEWANN, Geschichte 2, 383.

${ }^{48}$ Központi Statisztikai Hivatal, 1949, 11.
}

treibung der Deutschen in Ungarn für Unrecht und drückte sein Beileid für die Angehörigen der dabei Verstorbenen und sein Mitgefühl für alle Betroffenen aus. ${ }^{49}$ Die Opfer der Zwangsarbeit in der Sowjetunion bekamen finanzielle Entschädigung ${ }^{50}$ und es erfolgte eine schrittweise Auseinandersetzung mit den Vertreibungen. Ungarn bekannte sich zu seiner Verantwortung und das ungarische Parlament erklärte 2012 den 19. Januar (am 19. Januar 1946 begann die Vertreibung) zum Gedenktag der Verschleppung der Ungarndeutschen. ${ }^{51}$

Ein wichtiger Schritt in der juristischen Aufarbeitung war, als 2007 das ungarische Verfassungsgericht in einem Beschluss erklärt hat, dass die Verordnungen 12.330/1945 ME. und 12.200/1947 Korm. verfassungswidrig geworden sind, folglich nicht anwendbar sind, und auf ihnen keine Ansprüche mehr gegründet werden können. 52

\section{Zusammenfassung}

Wie oben dargestellt, änderte sich die Regelung über die Rechtstellung und Rechte der Ungarndeutschen in der ersten Hälfte des 20. Jahrhunderts relativ oft, sie folgte immer den veränderten politischen Zielen und Vorgaben. Die betroffene ungarndeutsche Bevölkerung war eher Objekt als Subjekt der Vorgänge, deren Meinung wurde kaum gefragt, ihre politische Interessenvertretung während der ganzen Epoche schwach, vielfach waren sie über die Rechtsnormen, die die Rechte der nationalen Minderheiten betrafen, nicht oder nicht hinreichend informiert. Die Ereignisse nach dem Zweiten Weltkrieg halbierten die ungarndeutsche Ge-

\footnotetext{
49 Parlamentsbeschluss Nr. 34/1990. OGY.

${ }^{50}$ Gesetz Nr. 29 von 1997 i.V.m. Verordnung 267/2000. Korm.

${ }^{51}$ Parlamentsbeschluss Nr. 88/2012. OGY.

${ }^{52}$ Beschluss des Verfassungsgerichts 92/2007. (XI. 22.) AB, in: Magyar Közlöny 159 (2007).
} 
meinschaft und trugen zur fortschreitenden Assimilation der in Ungarn Verbliebenen bei. In der politischen und juristischen Aufarbeitung der Geschehnisse ist Ungarn wohl am weitesten unter den an der Vertreibung beteiligten Staaten, dies kann das Geschehene zwar nicht ungeschehen machen, ermöglicht aber ein friedliches Zusammenleben, und ist in der ganzen Region als beispielhaft zu bezeichnen.

\section{Korrespondenz}

Dr. Dóra FREY

Andrássy Universität Budapest

Pollack Mihály tér 3.

$\mathrm{H}-1088$ Budapest

dora.frey@andrassyuni.hu

ORCID-Nr.: 0000-0002-7075-3185

\section{Abkürzungen:}

$\mathrm{AB} \quad$ Alkotmánybíróság (Verfassungsgericht)

BM Belügyminisztérium (Innenministerium)

Korm. Kormány (Regierung)

ME Miniszterelnök (Ministerpräsident)

MT Minisztertanács (Ministerrat)

OGY Országgyűlés (Landesversammlung)

Siehe auch das allgemeine Abkürzungsverzeichnis: [http://www.rechtsgeschichte.at/media/abk.pdf]

\section{Literaturverzeichnis}

Sándor BALOGH, Levente SiPOS, József KARDOs (Hgg.), A magyar állam és a nemzetiségek. A magyarországi nemzetiségi kérdés történetének jogforrásai 1948-1993 (Budapest 2002).

Béla BELLÉR, Az ellenforradalmi rendszer első éveinek nemzetiségi politikája (1919-1922), in: Századok 6 (1963) 1279-1318.

DERS., A Magyar Népköztársaság és a Tanácsköztársaság nemzetiségi kultúrpolitikája, in: Történelmi Szemle 1-2 (1969) 1-22.

Jakob BLEYER, A hazai német kisebbség kérdéséről, in: Magyar Szemle 9 (1933) 72-77.

Zoltán CZIBULKA, Ervin HeINZ, Miklós LAKATOS (Hgg.), A magyarországi németek kitelepítése és az 1941. évi népszámlálás (Budapest 2004).
Holger FISCHER, Oszkár Jászi und Mihály Károlyi. Ein Beitrag zur Nationalitätenpolitik der bürgerlichdemokratischen Opposition in Ungarn von 1900 bis 1918 und ihre Verwirklichung in der bürgerlich-demokratischen Regierung von 1918 bis 1918 (München 1978).

Gizella FöGLEIN, Etnicum és educatio. A magyarországi nemzetiségek és alsó fokú oktatásuk állami szabályozása 1945-1985 (Budapest 2006)

Miklós FÜZES (Hg.), Modern rabszolgaság „Malenkij Robot". Magyar állampolgárok a Szovjetunió munkatáboraiban 1945-1949 (Budapest 1990).

DERS., András ÚJvÁRI, Mentsvár. A pécsi 8/III. pótzászlóalj németajkú önkéntesei 1944-ben (Pécs 1996).

Gusztáv GrATZ, Bleyer Jakab, in: Magyar Szemle v. 4. 1. 1936, 12-16.

Vendel Hambuch (Hg.), Jakob Bleyer. Ein Leben für das Ungarndeutschtum (1874-1933) (Budapest 1994).

László KATUS, Nemzetiségi adatsorok a dualizmus korában, in: Ferenc GLATZ (Hg.), Magyarok a Kárpát-medencében (Budapest 1989) 193-200.

Központi Statisztikai Hivatal (Hg.), 1949. Évi népszámlálás, $\mathrm{Bd}$. 9: Demográfiai eredmények (Budapest 1950).

Barna Mezey (Hg.), Magyar alkotmánytörténet (Budapest 2003).

Ignác RoMsICS, Az 1947-es párizsi békeszerződés (Budapest 2006).

András Rozs, A népinémet mozgalom erősödése az Anschlusst követően a Dunagőzhajózási Társaság pécsvidéki bányatelepein, in: László SzITA (Hg.), Baranyai Történetírás 1990/1991. A Baranya Megyei Levéltár Évkönyve (Pécs 1992) 395-410.

István SCHLETT, Eötvös József (Budapest 1987).

Gerhard SEEWANN, Geschichte der Deutschen in Ungarn, Bd. 2: 1860-2006 (Marburg 2012).

DERS., A németek Kelet-Európából való elűzésének tipológiai sajátosságai, in: István LENGVÁRI, József VONYÓ, Népek együttélése Dél-Pannóniában. Tanulmányok Szita László 70. születésnapjára (Pécs 2003) 343-354.

Gernot SEIDE, Die Deutschen in Ungarn zwischen den beiden Weltkriegen, in: Ungarn Jahrbuch 6 (19741975) 148-161.

Norbert SPANNENBERGER, A magyarországi Volksbund Berlin és Budapest között 1938-1944 (Budapest 2005).

DERS., Nemzetiszocialista népcsoportpolitika DélkeletEurópában a II. világháború kitöréséig, in: István LENGVÁRI, József VONYÓ, Népek együttélése Dél- 
Pannóniában. Tanulmányok Szita László 70. születésnapjára (Pécs 2003) 411-433.

Tamás STARK, „Malenki robot“. Magyar kényszermunkások a Szovjetunióban, in: Kisebbségkutatás 1 (2005) 65-75.

Lóránt TiLKovszKY, A Bleyer-portré problematikus vonásai, in: Történelmi Szemle 3-4 (1993) 259-277.

DERS., Német nemzetiség - magyar hazafiság. Tanulmányok a magyarországi németség történetéből (Pécs 1997).

DERS., SS-toborzás Magyarországon (Budapest 1974).

DERS., Teufelskreis. Die Minderheitenfrage in den deutsch-ungarischen Beziehungen 1933-1938 (Budapest 1989).
Ágnes TóTH, Telepítések Magyarországon 1945-1948 között. A német kitelepítés, a belső népmozgások és a szlovák-magyar lakosságcsere összefüggései (Kecskemét 1993).

DIES., Földreform, internálás, belső telepítés Magyarországon 1945 után, in: Barátság 3 (2006) 5018-5023.

Zsolt VITÁRI, Új kisebbségpolitika ellenérzésekkel? Etnopolitikai irányváltások Magyarországon 1918 és 1920 között, in: Krisztina ScHLACHTA, Gábor BÁNKÚTI, József VONYÓ (Hgg.), Sed intelligere. Tanulmányok a hatvanöt éves Gyarmati György tiszteletére (Pécs 2016) 181-199.

György ZIELBAUER, Die Verschleppung ungarländischer Deutscher 1944/45. Erste Station der kollektiven Bestrafung (Budapest 1990). 